\title{
T-Cell Large Granular Lymphocytic Chronic Leukemia: Treatment Experience with Cyclosporine
}

\author{
Guran Catalina ${ }^{2 *}$, Ilie Alina ${ }^{1}$, Oros Dacian Nicolae ${ }^{2}$, Calamar Popovici Despina ${ }^{2}$ and Ionita Ioana ${ }^{2}$ \\ ${ }^{1}$ Hematology Department, Emergency Municipal Hospital Timisoara, Romania \\ ${ }^{2}$ Department of Internal Medicine 1 - Hematology, “Victor Babes” University of Medicine and Pharmacy Timisoara, Romania, Eftimie Murgu Sq. no. 2, \\ 300041, Timisoara, Romania
}

Submission: July 15, 2020; Published: July 23, 2020

*Corresponding author: Guran Catalina, Department of Internal Medicine 1 - Hematology, "Victor Babes" University of Medicine and Pharmacy Timisoara, Romania, Eftimie Murgu Sq. no. 2, 300041, Timisoara, Romania

Abstract

The patient presented herself at the hospital in June 2018 with severe anemia $(\mathrm{Hgb}=5,4 \mathrm{~g} / \mathrm{dl})$, mild leucopenia $\left(\mathrm{Le}=3980 / \mathrm{mm}^{3}\right)$ and grade III neutropenia $\left(\mathrm{Ne}=557 / \mathrm{mm}^{3}\right)$. She underwent bone marrow biopsy and flow cytometry which established the diagnosis of Large Granular T-cell Leukemia. She was given treatment with Ciclosporine $50 \mathrm{mg} 6 \mathrm{cps} /$ day, which was reduced at $3 \mathrm{cps} /$ day because the patient developed pancytopenia at $6 \mathrm{cps} /$ day. The blood count test after 6 months of treatment was greatly improved: she obtained normal levels of hemoglobin and platelets, with the persistence of asymptomatic neutropenia. At the one-year follow-up we had an adequate response, but at the 18 months follow-up she has developed mild pancytopenia, which leads us to believe that she is relapsing under the Ciclosporine treatment.

Abstract: T- LGL; Ciclosporine

\section{Introduction}

T-cell Large Granular Lymphocytic Chronic Leukemia is lymphoproliferative disorder characterized by enlarged lymphocytes, containing noticeable granules. It was described as a clonal disorder involving the invasion of marrow spleen and liver. Recurrent infections are usually seen in patients with T-LGL leukemia, associated with neutropenia [1]. We can also see anemia, splenomegaly and autoimmune disease, particularly rheumatoid arthritis. We are going to present a case of T-LGL chronic leukemia followed by our team over a period of 18 months.

\section{Case Report}

The patient, G.A., 68 years, female, with rheumatoid arthritis as associated risk factors, first presented herself at the Emergency Department in June 2018 with severe fatigue, dizziness, tegumentary pallor. She was admitted with severe anemia $(\mathrm{Hgb}=5,4 \mathrm{~g} / \mathrm{dl})$, was given blood transfusions. The abdominal echography showed mild hepatomegaly and splenomegaly. In July 2018 she was again admitted with severe anemia and grade III neutropenia, was given blood transfusions and underwent a bone marrow aspirate, which was inconclusive. She was recommended a bone marrow biopsy which was unavailable in the Clinic she was admitted to, there fore the patient presented herself in the Hematology Clinic of the Municipal Emergency Hospital in Timisoara, in November 2018.

She underwent several tests, a bone marrow biopsy (Conclusions: Medullar infiltration $\sim 15-17 \%$, with small T-cell with a phenotype of large granular lymphocytes- T-cell large granular lymphocytic leukemia. Erytoblastic hyperplasia and moderate neutropenia) correlated with flow cytometry (Phenotype: 46\% Lymphocytes T CD3+, CD8+, TCRgd-, CD5 weak positive) in November 2018, which established the diagnosis of T-cell large granular lymphocytic leukemia [2,3]. Due to pharmaceutical limitations (oral Fludarabine and Cyclophosphamide being very difficult to obtain in our country), the patient was started with oral Cyclosporine 6cps/day in January 2019, associated with prophylactic antibiotics. Due to worsening of the blood count test, the dose was reduced at $3 \mathrm{cps} /$ day. She maintained a level of $\mathrm{Hg}$ $\mathrm{b} \sim 10 \mathrm{~g} / \mathrm{dl}$, grade II/ III asymptomatic neutropenia, and no thrombocytopenia (Figure 1).

In August 2019 she presented with $\mathrm{Hgb}<10 \mathrm{~g} / \mathrm{dl}$, which allowed us to recommend Erythropoietin treatment [4]. After one month of erythropoietin she obtained $12 \mathrm{~g} / \mathrm{dl}$ and maintained a level over $10 \mathrm{~g} / \mathrm{dl}$ of hemoglobin. At the one year follow up the pa- 


\section{Cancer Therapy \& Oncology International Journal}

tient presented herself with grade II asymptomatic neutropenia, without anemia or thrombocytopenia. She was considered in a partial remission and the Cyclosporine treatment was continued with $3 \mathrm{cps} /$ day, associated with prophylactic antibiotics (Figures
$2 \& 3)$. We are now at the 18 months follow-up, when the patient presented herself with pancytopenia, $\mathrm{Hgb}=10,3 \mathrm{~g} / \mathrm{dl}, \mathrm{Ne}=1250 /$ $\mathrm{mm}^{3}, \mathrm{Plt}=118.000 / \mathrm{mm}^{3}$. We are currently suspecting a relapse and are re-evaluating her as such.

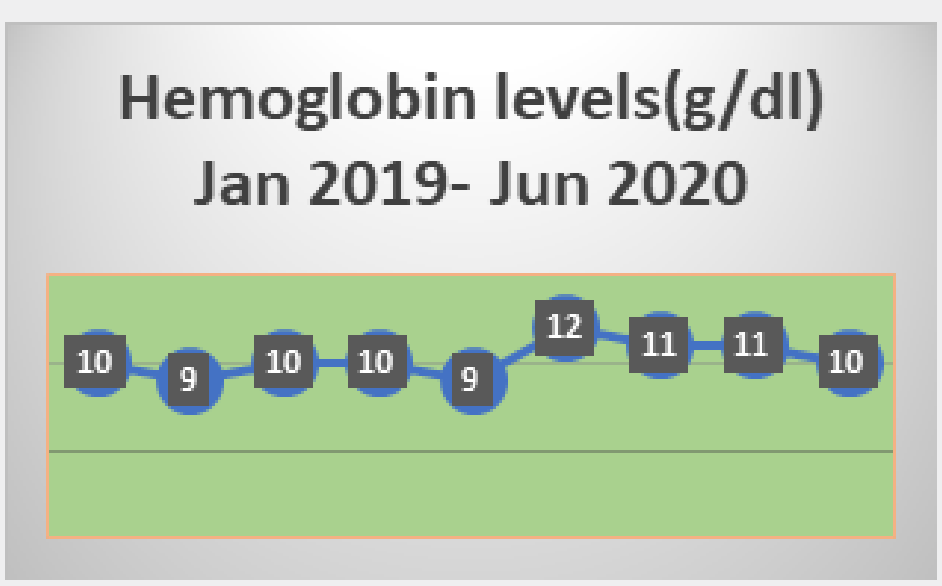

Figure 1:Hemoglobin levels during the follow-ups.

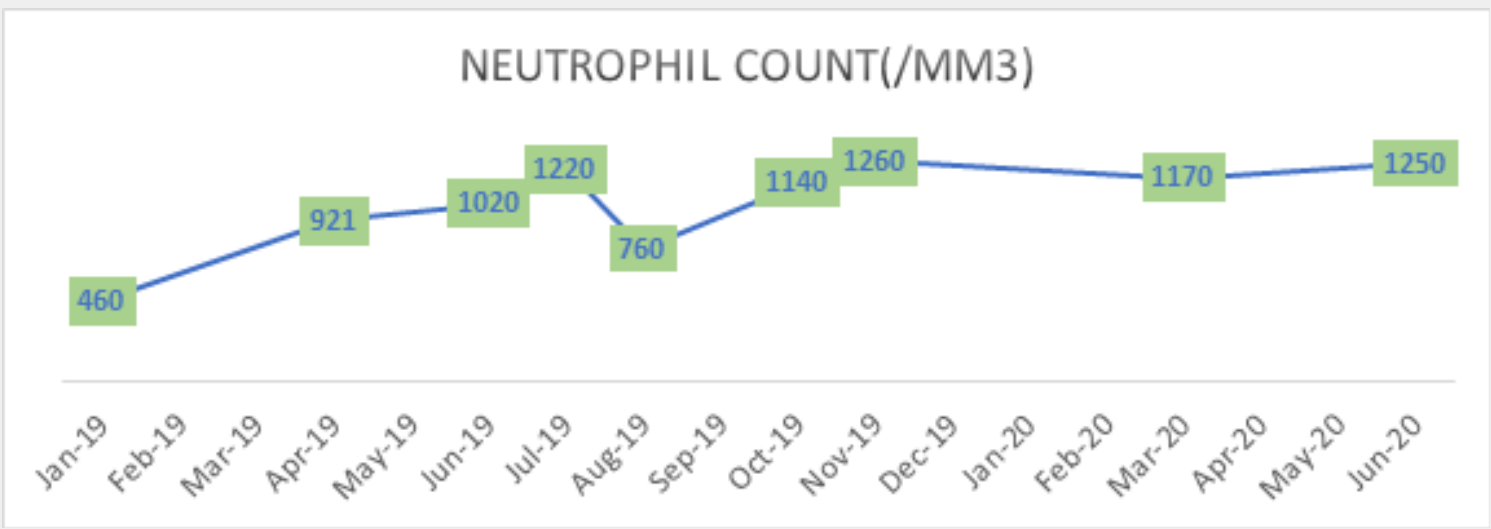

Figure 2: Graphic representation of neutrophil count.

\section{Platelet count (/mm3)}

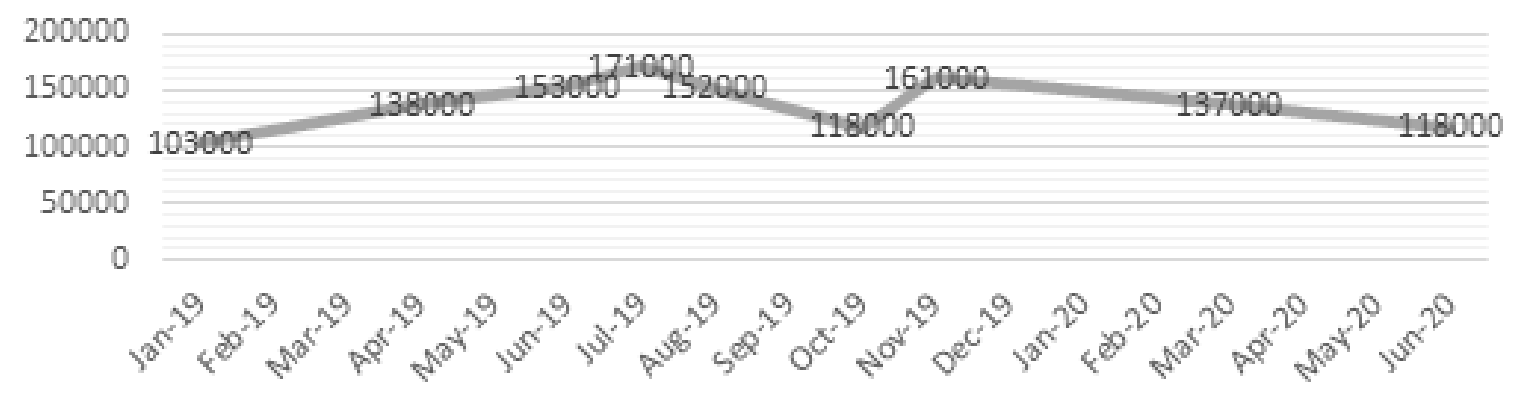

Figure 3: Graphic representation of the evolution of the patient platelet count. 


\section{Cancer Therapy \& Oncology International Journal}

\section{Conclusion and Discussion}

i. The apparent response at this patient is of 18 months, with a very good quality of life, although the blood count evolution was uneven.

ii. She required only a month of supportive treatment after diagnosis, without the need of systemic treatment, lowering the risk for complications.

iii. The quality of life during this time was very good, with no clinical symptoms and no complications. This was the goal we (the patient and medical team) set when starting treatment. For now, the goal has been achieved.

iv. The patient had no need for blood transfusions after the treatment was started, which led to a better compliance to treatment and lower costs. Of course, the lowering of costs of treatment is a factor that we like to keep in mind, but maybe not a decisive factor when deciding on the treatment we give a patient. v. We never achieved a complete hematological response; we need to continue the monitoring of the patient to be able to have a definite relapse diagnosis. Although, until we have established a relapse diagnosis, taking into consideration the current situation with the COVID-19 pandemic and the clinical state of the patient, we are taking into consideration on continuing with the current treatment for the moment.

\section{References}

1. Thierry Lamy, Aline Moignet, Thomas P Loughran Jr (2017) LGL leukemia: from pathogenesis to treatment, Thierry Lamy, Aline Moignet, Thomas P. Loughran, Jr, Blood 129(9): 1082-1094.

2. Thierry Lamy, Thomas P Loughran, Jr (2011) How I treat LGL leukemia. Blood 117(10): 2764-2774.

3. Neha Gupta, Suman Kumar, Gurdeep Singh, Wg Cdr, Atoshi Basu, et al (2015) Adult T cell large granular lymphocytic leukemia. Med J Armed Forces India. 71(Suppl 1): S272-S275.

4. Aline Moignet, Thierry Lamy (2018) Latest Advances in the Diagnosis and Treatment of Large Granular Lymphocytic Leukemia. Am Soc Clin Oncol Educ Book 38: 616-625.

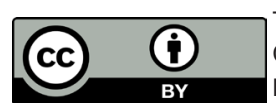

This work is licensed under Creative Commons Attribution 4.0 License DOI:10.19080/CTOIJ.2020.16.555943 